\title{
Optimal Provision of Global Public Goods under Uncertainty: Strategic Transfers and Reservations in a Multilateral Treaty Obligation*
}

\author{
Jin Kim**, Seung-jin Shim***
}

\begin{abstract}
This paper studies the formation and management of an international entity for promoting the provision of global public goods in a setup of international treaties on concessions and reservations. Based on the so-called Vienna Convention by which there can be a discrepancy between the original treaty obligation and the ratified one in the multi-lateral treaty implementation, we construct two-stage mechanisms for international treaties on global public goods; the concession stage and the ratification stage with reservations. We explicitly analyze the optimal reservation levels from the optimal mechanism when the countries face asymmetric information on the preference parameters to global public goods. Specifically, we characterize the environments where the optimal mechanism with dominant-strategy incentive-compatibility and ex-post participationconstraint in the literature of mechanism design exists. The result shows that the sophisticatedly calculated transfers in the principal of quid pro quo control the international concessions and reservations.
\end{abstract}

Key Words: Global Public Goods, Concessions, Reservations, Transfers,Multi-lateral Treaty

JEL codes: H87, K33, F53, C72

* This research was supported by Kyungpook National University Research Fund, 2014.

** Main Author: Department of Economics, Dongduk Women's University, Korea. Tel: +81-2-940-4442, e-mail:jkim@dongduk.ac.kr

*** Corresponding Author: School of Economics and Trade, Kyungpook National University, Korea. Tel: +81-53-950-5437, e-mail: shimsj@mail.knu.ac.kr 


\section{Introduction}

The importance of global public goods (GPGs) has recently grown in various fields such as environments, diseases, and financial crises in the realm of the world. In particular, the demand for GPGs has been increasing apace with globalization. ${ }^{1)}$ We have also recognized that net benefits from GPGs get large and pervasive. In some extreme cases of GPGs, as Sandler (1997) points out, the benefits disperse worldwide; for instance, efforts to curb global warming, to reduce ozone-depleting chlorofluorocarbon (CFC) emissions or CO2-emissions, to map the human genomes, or to preserve the earth's biodiversity. However, GPGs always have the characteristics of having free rider problems and underproduction.

International cooperative arrangement to supplying GPGs is normally codified in international treaties, as Barrett (2001) presents. We observe that before the Vienna Convention in 1969 it used to be conventional that, in order to promote the agreement and stability of international treaties, the countries involved are not allowed to consider voluntary reservations or side payments. However, there has been a dramatic change in the international conventions on the introduction of side payments and voluntary reservations. Now it is not a new phenomenon that the countries involved would like to think of side payments or transfers such as in the international environmental agreements, ${ }^{2}$ ) or voluntary reservations such as in the 1969 Vienna Convention.3)

This paper investigates international treaties on the provision of GPGs such as international environment agreements within the viewpoint of concessions, reservations, and transfers formally in the framework of mechanism design. The focal point of this study is on informational incompleteness under which we model the situation where the countries involved would like to set up an optimal mechanism of international treaties when there is informational asymmetry on the preference to GPGs among them. Thus, we model the situation where the countries involved may do strategic actions (for concessions, reservations, and transfers) in the given games consisting of the mechanism (as the international treaty) and any realized state (of each country's private information on the preference to GPGs).

Specifically, we are concerned about the conventional two-stage approach for the agreement and revision of the international treaty on the $\mathrm{CO} 2$-emissions reduction. In the Kyoto Protocol we find a separation between the agreement and ratification as in the

\footnotetext{
1) See Sandler (1997), Kaul, Grunderg and Stern (1999), and Ferroni and Mody (2002).

2) Barrett (2001) studies the feature of side payments in the international environmental agreements.

3) Refer to Fon and Parisi (2003) for reservations.
} 
general cases of the international treaties. That separation improves the speed of the agreement at the outset and concretely incorporates the ratification of the reservation level later in each country.

For the purpose of analysis, therefore, we will consider two-stage mechanisms; the first stage for concessions and the second one for ratification/reservations. ${ }^{4)}$ At the stage of concessions, the countries involved meet and form a treaty for the amount of GPGs and each country's contribution limit. At the stage of reservations or ratifications, each country ratifies the level of concessions with reservation modifications.

As sure, at the transition from the concession stage to the ratification stage there would be a kind of information refinement on the uncertainty. We observe that for the case of CO2-emission reduction, the preference for the global contribution to the reduction become clearer as time goes. Therefore, we may think the type set of the preference parameter gets refined as time goes.

Fon and Parisi(2003) analyze the optimal level of reservations mainly in a two-country model. Referring to the role of the Vienna Convention in 1969 on modern treaty-making, they characterize treaty ratifications in a bilateral treaty within the framework of Prisoners' Dilemma games. They show the possibility of voluntary reservations as a Nash equilibrium in the setup of complete information in the sense that every country's benefit and cost parameters are common knowledge.

Our study departs from the analysis of Fon and Parisi(2003) in two ways. First of all, we admit the incompleteness of information in that every country's benefit parameters are private information.5) Secondly, we will use the well-developed analytical tool of mechanism design. Especially, we think the ex post participation constraint should be required in international treaties. Thus, we will use dominant-strategy incentive-compatible mechanisms in the literature of mechanism design.

Based on the convention that there is a discrepancy between the original treaty obligation and the ratified one in the multi-lateral treaty implementation, we select the ratification levels of the countries as the choice variables in an economic model. We explicitly analyze the optimal reservation levels of the optimal mechanism when countries face asymmetric information on the ratification levels. Specifically, we characterize the environments where the optimal mechanism with incentive compatibility and participation constraint in the literature of mechanism design exists.

4) Fon and Parisi (2003) analyze the characterization of the Vienna Convention in the viewpoint of law economics. They introduce an economic model to analyze the mechanism of concessions and reservations in the history of international treaties.

5) We delay the analysis for the cost uncertainty later and assume that the unit cost from the private good to the contribution is the same over countries. 
Theoretically, by using the expenditure minimization behavior, ${ }^{6)}$ we can formalize indirect utility functions as valuation functions in game theory, to which we add mechanisms with message spaces and the decision rules of reallocations and monetary transfers. Based on the mechanism design theory of the Groves mechanisms, we analyze a possibility of dominant-strategy incentive mechanisms with transfers in the case of preference uncertainty.

We propose an alternative division method of the potential surplus from the international treaty over GPGs in contrast to the literature of the Groves mechanisms. Our proportional division of the surplus admits a kind of fairness in that the country with higher preference parameter values may receive larger surplus in a proportional way eventually in average. We finally characterize a necessary and sufficient condition for the existence of that mechanism. ${ }^{7}$ )

This paper consists of 5 sections. The second section introduces our basic model of incomplete information on the preference of the countries to GPGs. Section 3 lays out the contents of the literature of the Groves mechanisms, constructs incentive mechanisms, and supplies a characterization proposition for the optimal mechanism with dominant-strategy incentive compatibility and ex post participation constraint. Section 4 discusses the meaning of the proposition in a two-country case and deals with the implication of our result into the international treaty practices. Section 5 concludes.

\section{Model}

We assume that there are $n$ countries being interested in forming an international treaty on the contributions to a global public good (GPG). Specifically, we may think about the international treaty on the CO2-reduction activities. Thus, there are two goods in the world in our model; a global public good and a private good. According to the tradition in the literature of global public goods, we restrict our attention to quasi-linear welfare functions; $u^{i}\left(x_{i}, G\right)=x_{i}+\theta_{i} \operatorname{In}(G)$ for country $i$ where $\theta_{i}$ is the degree of preferring GPG to the private good and $x_{i}$ is the consumption level of the private good for country $i$. Here, the amount G of GPG consists of each country $i$ 's contribution $z_{i}$ of GPG, which is transformed from the private good $x_{i}$; Thus, $G=z_{1}+z_{2}+\cdots+z_{n}$. By assuming that $x_{i}$ is the numeraire good, country $i$ 's budget constraint would be $x_{i}+p z_{i}=Y_{i}$, where $p$

6) See Ihori $(1994,1996)$ for its development in the context of international public goods.

7) Laffont and Martimort (2005) recently analyze the design of incentive mechanisms for the provision of transnational public goods under asymmetric information among countries. 
denotes the common unit cost for producing $z_{i}$ and $Y_{i}$ denotes the income of the country $i$.

We assume that $p$ and $Y_{i}$ 's are given fixed and regarded as public information in the sense of common knowledge, and that $\theta_{i}$ 's are private information called $i$ 's types. Let $\Theta_{i}=\left[\theta_{\underline{i}}, \bar{\theta}_{i}\right]$ be the set of $i$ 's types with $\theta_{\underline{i}}>0$. Let us decompose the state set $\Theta=\prod_{i} \Theta_{i}$ into $n$ subsets; for each $i, \Theta^{*_{i}}=\left\{\theta \in \Theta \mid \theta_{i}=\max _{j} \theta_{j}\right\}$ is the set of the states where country $i$ has the highest preference parameter.

We consider two stages of an international treaty in a strategic sense; the first stage for concessions and the second for reservations from ratifications. At the stage of concessions, the countries meet and form a treaty for the amount of $\mathrm{G}$ and each nation's contribution limit. At the stage of reservations, each country ratifies the level of concession with a possible reservation modification. Our assumption of two-stage treaty-formation seems to be natural in international treaties.

At the stage of concessions in a treaty, the countries involved would like to join a treaty on providing GPGs as many as possible. However, the types of countries are not realized at this moment. The important thing to be done by the countries at this stage, then, is how to take a kind of uncertainty about the degree of preferring GPG.

There are two approaches to that uncertainty. On the one hand, the Bayesian approach uses the distribution function on type sets. On the other hand, the Groves approach in the sense of dominant strategy incentives does not use any kind of detailed information on the distribution function. In the international society where there is lack of distribution function, the Groves approach would be selected. That means that at any case the international treaty should provide the optimal level of GPG and maintain any kind of monetary transfers for incentives.

By assuming that each country $i$ takes the very conservative stance for uncertainty at the outset of an agreement, an international treaty would be formalized based on the assumed case $\bar{\theta}_{i}$ of the highest degree of preferences for GPG. Then, the Pareto optimal allocation at $\bar{\theta}_{i}$ could be decided as the concession ceiling for country $i$. Formally, for each $i, \overline{V_{i}}=\frac{\bar{\theta}_{i}}{p}$ would be the concession ceiling and $\bar{x}_{i}=Y_{i}-\bar{\theta}_{i}$ would be the minimal consumption level of private good. Thus, $\bar{G}=\frac{1}{p} \sum_{j} \bar{\theta}_{j}$ would be the maximal level of GPG at the very conservative stance. We here assume that the level of concessions in an international treaty, determined as $\overline{V_{i}}(\theta)=\frac{\bar{\theta}_{j}}{p}$ for any country $j$, is prepared for any 
possible future 'state of nature' $\theta$ of the world. Of course, the countries involved know that that level is functioned as the maximal level of concessions and that they will later have a chance to having reservations from that level at the stage of ratification. We observe that the concession levels in our setup are heterogeneous among countries. In reality, there is diversity on the concession levels for the $\mathrm{CO} 2$-reduction contributions over countries, especially between the developed and the developing countries.

At the stage of ratification, the types of countries are realized and the mechanism based on the international treaty is operated. The information on the types is revealed at this moment. However, the information on the preferences is private, thus there may be asymmetry in information. It is well known that there is a free rider problem in that situation. There is an incentive to understate the importance of the GPG in order to reduce the contribution for the GPG because of externality and asymmetric information. Since each country $i$ knows her preference parameter $\theta_{i}$ and is only aware of the distribution of the other country's preference parameters, one of the important roles of the international entity would be how to obtain the true information about $\theta_{i}$ 's from the member countries through an incentive compatible mechanism.

In order to implement the first-best allocation, we assume that the international entity use the Groves mechanism in the literature of mechanism design. Thus, we assume that the countries involved make the international entity install an international agency that collects the reports on types from the member countries and decides allocations and transfers for the member countries.

The optimal level of ratification through the legistrative process in each country would be calculated if there is complete information on the preference parameter $\theta$. In a sense, the optimal level would be obtained as a byproduct from the Pareto optimal allocation in the case of complete information. The Pareto allocation is that, for each $i$ at $\theta, z_{i}(\theta)=\frac{\theta_{i}}{p}$ and $x_{i}(\theta)=Y_{i}-\theta_{i}$, thus $\mathrm{G}(\theta)=\frac{1}{p} \sum_{i} \theta_{i}$. Let A be the set of all the feasible outcomes with $\left(x_{1}, \cdots x_{n}, z_{1}, \cdots, z_{n}\right) \in A$. Then, by using indirect utility functions from the abovementioned method, we may set up a valuation function $v_{i}\left(\bullet, \theta_{i}\right)$ over $A$ for each type $\theta_{i}$. Specifically, the payoff of country $i$ with type $\theta_{i}$ from the reports $\hat{\theta}$ is

$$
v_{i}\left((x(\hat{\theta}), z(\hat{\theta})), \theta_{i}\right)=\theta_{i} \operatorname{In}\left(\frac{1}{p} \sum_{k} \hat{\theta_{k}}\right)+Y_{i}-\hat{\theta}_{i}
$$

We can verify that the valuation functions in (1) satisfy the convexity condition of 
Holmström (1979). Thus, by following Makowski and Mezzetti (1994), we can apply the Groves mechanism into our setup.

Before the formal analysis of the Groves mechanism in Section 3, we now explain why we focus on the Groves mechanism. As in the case of $\mathrm{CO} 2$-emission reductions, the countries involved face uncertainty on preference parameters without any kind of knowledge on distribution functions. In addition, the international society should concern about the worst case of uncertainty at the very conservative stance. Therefore, we prefer the Groves mechanisms to Bayesian mechanisms in our international treaty-making environment.

We will develop the conservative concessions at the first stage with a possibility of reservations at the second stage. To do this purpose, we can think several alternative mechanisms such as (i) the status quo without a certain mechanism, (ii) a Nash equilibrium without transfers, and (iii) an optimal reservation mechanism with transfers. "No mechanism" in (i) means that there is no explicit and intentional concern on an international entity in the international society. Then the concessions given already are to be just an empty promise without any commitment.

Nash equilibrium without transfers in (ii) is a la Ihori $(1994,1996)$. Even though there is an improvement on the level of international treaty, that level out of Nash equilibrium is below the optimal level.

Optimal reservation mechanism with transfers in (iii) would be constructed a la Groves and Loeb (1975). The international level of ratification is optimal and there are monetary transfers among the member countries. Section 3 will show the formal analysis of the mechanism design in the context of international treaties.

\section{Incentive Mechanisms under Preference Uncertainty}

A direct mechanism8) is denoted by $\Theta,<s, t>$ where $\Theta$ is the message space of the type reports and $\langle s, t\rangle$ is an outcome function. The outcome function consists of a decision rule $s: \Theta \rightarrow A$ and a transfer scheme $t=\left(t_{1}, \cdots t_{n}\right)$ with $t_{1}: \Theta \rightarrow \mathrm{R}$. We will abuse the notation $\langle s, t\rangle$ for a direct mechanism. Given $\langle s, t\rangle$, the payoff of country $i$ with type $\theta_{i}$ from a report composite $\hat{\theta}$ is $v_{i}\left(s(\hat{\theta}), \theta_{i}\right)+t_{i}(\hat{\theta})$ where $v_{i}$ is defined as in (1).

The global gain function from the Pareto allocation is

8) See Dasgupta, Hammond, and Maskin (1979). 


$$
V(\theta)=\sum_{i} v_{i}\left((x(\theta), z(\theta)), \theta_{i}\right)=\left[\sum_{i} \theta_{i} 1 n\left(\frac{1}{p} \sum_{k} \theta_{k}\right)+\sum_{i} Y_{i}-\sum_{i} \theta_{i}\right]
$$

After a direct mechanism is installed and a true state $\theta$ is realized, the countries face a direct revelation game where each member country submits her type to the international entity and the international entity decides the allocation based on the submitted type reports.

A mechanism $\langle s, t\rangle$ is dominant-strategy incentive compatible (DSIC) if every country has the incentive to report her own type honestly regardless of the others' report schemes at any state, i.e., for all $i$, for all $\theta_{-i}$, for all $\theta_{i}$, and for all $\theta^{\prime}{ }_{i}$,

$$
v_{i}\left(s\left(\theta_{-i}, \theta_{i}\right), \theta_{i}\right)+t_{i}\left(\theta_{-i}, \theta_{i}\right) \geq v_{i}\left(s\left(\theta_{-i}, \theta_{i}^{\prime}\right), \theta_{i}\right)+t_{i}\left(\theta_{-i}, \theta_{i}^{\prime}\right)
$$

A decision rule s is outcome-efficient if $\sum_{i} v_{i}\left(s(\theta), \theta_{i}\right)=V(\theta)$ for all $\theta$, that is, if it always realizes the global gain in (2). A mechanism $\langle s, t\rangle$ is a first-best dominant-strategy mechanism if it is outcome-efficient and dominant-strategy incentive compatible.

Since our setup satisfies the convexity condition in Holmström (1979), we can use his result that a mechanism is a first-best dominant-strategy if and only if it is a Groves mechanism. Following Makowski and Mezzetti (1994), then, we can define the participation charge on country $i$ at state $\theta$ as the difference of $i$ 's payoff in (3) from the global gain in $(2) ; h_{i}(\theta) \equiv V(\theta)-v_{i}\left(s(\theta), \theta_{i}\right)-t_{i}(\theta)$ for all $\mathrm{i}$ and $\theta$. As well known, a mechanism $\langle s, t>$ is a Groves mechanism if it is outcome-efficient and its participation charge $h_{i}\left(\theta_{-i}\right)$ on country $i$ is independent of $i$ 's type for each $i$. Then, country $i$ 's payoff from the participation into a Groves mechanism at state $\theta$ is

$$
v_{i}\left(s(\theta), \theta_{i}\right)-t_{i}(\theta)=V(\theta)-h_{i}\left(\theta_{-i}\right)
$$

Since each country's participation charge is non-distortionary lump-sum in Groves mechanisms, there is no incentive for any country to lie in the direct revelation game. Thus, the truthful revelation of the type for any country is guaranteed in Groves mechanisms.

One simple Groves mechanism is a mechanism with zero participation charges; $h_{i}\left(\theta_{-i}\right)=0$ for all $i$ and for all $\theta$. Then each country's payoff would be equal to the global gain $V(\theta)$ at each $\theta$ by (4), we know that the zero-charge Groves mechanism incurs a deficit $t_{i}(\theta)=V(\theta)-v_{i}\left(s(\theta), \theta_{i}\right)$ for country $i$ at state $\theta$. The (ex ante) expected budget deficit for country $i$ in the zero-charge Groves mechanism is then 


$$
B_{i} \equiv E\left[V(\theta)-v_{i}\left(s(\theta), \theta_{i}\right)\right]=E\left[\sum_{j \neq i} \theta_{j} \operatorname{In}\left(\frac{1}{p} \sum_{k} \theta_{k}\right)+\sum_{j \neq i}\left(Y_{j}-\theta_{j}\right)\right]
$$

A mechanism $\langle s, t\rangle$ is ex post individual rational (EPIR) if its payoff is not negative for country $i$ at state $\theta$, that is, $\left.\left.v_{i}\left(s_{\left(\theta_{-i}\right.}, \theta_{i}\right), \theta_{i}\right)+t_{i}\left(\theta_{-i}, \theta_{i}\right) \geq 0.9\right)$

Then, by (4), we get $V(\theta) \geq h_{i}\left(\theta_{-i}\right)$ for country $i$ at state $\theta$. Now we may have the ceiling of the lump-sum charge for the international entity facing the EPIR condition. Since the international entity does not directly observe country $i$ 's type, the maximal amount that the international entity can charge on country $i$ without violating country $i$ 's EPIR condition is, by using (4), $c_{i}\left(\theta_{-i}\right) \equiv \min _{\theta_{i}}\{V(\theta)\}$ for all $\theta_{-i}$; That is, the minimal payoff given the other countries' reports. Then, the (ex ante) expected lump-sum charge without violating country $i$ 's EPIR condition is

$$
C_{i} \equiv E\left[c_{i}\left(\theta_{-i}\right)\right]=E\left[\sum_{j} \theta j \operatorname{In}\left(\frac{1}{p}\left(\sum_{k \neq i} \theta_{k}+\underline{\theta_{i}}\right)\right)+\sum_{k} Y_{k}-\sum_{k \neq i} \theta_{k}-\underline{\theta_{i}}\right]
$$

(5) and (6) might be interpreted as two edges of a 'benefit-charge' analysis in that for each country the international entity measures the benefit from the zero-charge Groves mechanism and levies the corresponding lump-sum charge for her.

In plain terms, an annoying problem in the literature of Groves mechanisms is how to fairly divide the expected surplus from the mechanism. For economic environments of smaller size than in the classical mechanism design area, Makowski and Mezzetti (1994) obtain a necessary and sufficient condition for the existence of the efficient dominant-strategy mechanism with EPIR and EABB; $\sum_{i} C_{i} \geq \sum_{i} B_{i}$.

We observe that their mechanism incurs the equal division of the surplus from the Groves mechanism into the member countries. However, in many cases, the equal division of the surplus from the Groves mechanism does not guarantee a kind of fair division especially when the conditions of the countries are not the same. For example, in the case of CO2-emission reductions in the UN Framework Convention on Climate Change (UNFCCC), the equal division of the potential surplus from the international agreement is both inadmissible and unfair. The current debate around the Kyoto Protocol is focused on how to fairly divide the future benefit from the agreement in the face value of the present cost burdens. As Burniaux et al (2009) clearly indicates, the current debate for the

9) We assume that the outside option payoff of any country $i$ at any state is exogenous and normalized to zero as usual in the literature of the international public goods. 
CO2-emission reductions is formalized in the so-called WITCH model ${ }^{10)}$ by using the non-cooperative game framework and the Nash equilibria in the dynamic WITCH model are pervasively considered. The fundamental question in Burniaux et al (2009) is how to divide the benefit of the model fairly so that the participation rate would be increased.

The equal division of the expected surplus in the mechanism of Makowski and Mezzetti (1994) could not be accepted among the participating member countries when the mechanism is installed because the equal division does not consider the difference among countries. In the case of $\mathrm{CO} 2$-emission reductions, there are lots of differences such as technologies, preferences, resources, and policies among nations. In particular, there is a trend to distinguish the developed countries from the developing ones. The main question would be how to incorporate the difference in countries into the international treaty about the $\mathrm{CO} 2$-emission reductions.

We introduce an alternative surplus-division method; a proportional division. While the equal division is related with ex ante budget balanced-ness (EABB), $E\left[\sum_{i}^{n} t_{i}(\theta)\right]=0$, the proportional division is related with a stricter condition of budget balanced-ness; zero expected net transfer.

A mechanism $\left\langle s, t>\right.$ is zero expected net transferred (ZENT) if $E\left[t_{i}(\theta)\right]=0$ for each $i$. Obviously, the ZENT condition is stronger than the EABB condition. While EABB ensures that there is no inflow of budget from outside in average, ZENT ensures that there is no unfair inflow or outflow of budget within the member countries in average. ZENT guarantees that each member country in average receives the potential net gain according to her status from the installation of the international treaty. Thus, we apply the proportional division according to the condition of ZENT.

As Makowski and Mezzetti (1994) obtain a necessary and sufficient condition $\sum_{i} C_{i} \geq \sum_{i} B_{i}$ for the existence of the efficient dominant-strategy mechanism with EPIR and $\mathrm{EABB}$, now we propose a necessary and sufficient condition for the existence of an efficient dominant-strategy mechanism with EPIR and ZENT as in Proposition 1.

Proposition 1: There exists an international entity which is first-best dominant-strategy incentive compatible (DSIC), ex post individual rational (EPIR), and zero-expected net-transferred (ZENT) if $E\left[c_{i}\left(\theta_{-i}\right)\right] \geq E\left[V(\theta)-v_{i}\left(s(\theta), \theta_{i}\right)\right]$ for all $i$.

10) For the details of the dynamic WITCH(World Induced Technological Change Hybrid) model, see Burniauz et al (2009). 
Proof: (If) Define a transfer scheme $t$ by $t_{i}(\theta)=V(\theta)-v_{i}\left(s(\theta), \theta_{i}\right)-c_{i}\left(\theta_{-i}\right)+K_{i}$ for all $i$ and $\theta$, where $K_{i}=E\left[c_{i}\left(\theta_{-i}\right)\right]-E\left[V(\theta)-v_{i}\left(s(\theta), \theta_{i}\right)\right] \geq 0$. Then, $\langle s, t\rangle$ is a Groves mechanism. It's trivial to check out EPIR and ZENT.

(Only if) By the result of Makowski and Mezzetti (1994), it suffices to show that $E\left[t_{i}(\theta)\right]=0$ for all $i$. By definition, $E\left[t_{i}\left(\theta_{i}\right)\right]=0$ for all $i$. Q.E.D.

The import of the proof in Proposition 1 is the sufficiency part. The proof still holds even when the type set of each member country is different and the distribution on it is diverse. In the setup of common type sets, the equal division of Makowski and Mezzetti (1994) and the proportional division of ours are the same, though. However, when the type sets are diverse among the member countries, our proportional division is dominant to their equal division in the sense that our proportional division is acceptable since it guarantees a kind of fairness in the international society.

\section{Implications}

\subsection{The Existence of Optimal Mechanisms}

The above conditions in Proposition 1 bring forth the range of the consumption level of non-GPG for the existence of the incentive mechanism in the two-country case; for EABB with (7) and for ZENT with (8), respectively.

$$
\begin{aligned}
& \overline{x_{1}}+\overline{x_{2}} \geq N \equiv E\left[\operatorname{In}\left[\left(\frac{\theta_{1}+\theta_{2}}{\left(\theta_{1}+\underline{\theta_{1}}\right)\left(\theta_{2}+\underline{\theta_{2}}\right)}\right)^{\theta_{1}+\theta_{2}}\right]\right] \\
& \overline{x_{i}} \geq N_{i} \equiv E\left[\operatorname{In}\left[\left(\frac{\left(\theta_{1}+\theta_{2}\right)^{\theta_{j}}}{\left(\theta_{j}+\underline{\theta_{j}}\right)^{\theta_{1}+\theta_{2}}}\right)\right]\right], \text { where } i, j=1,2 \text { and } i \neq j
\end{aligned}
$$

with $\overline{x_{i}} \equiv Y_{i}-\underline{\theta_{j}}$ being the maximum consumption level of non-GPG of country $i$. 


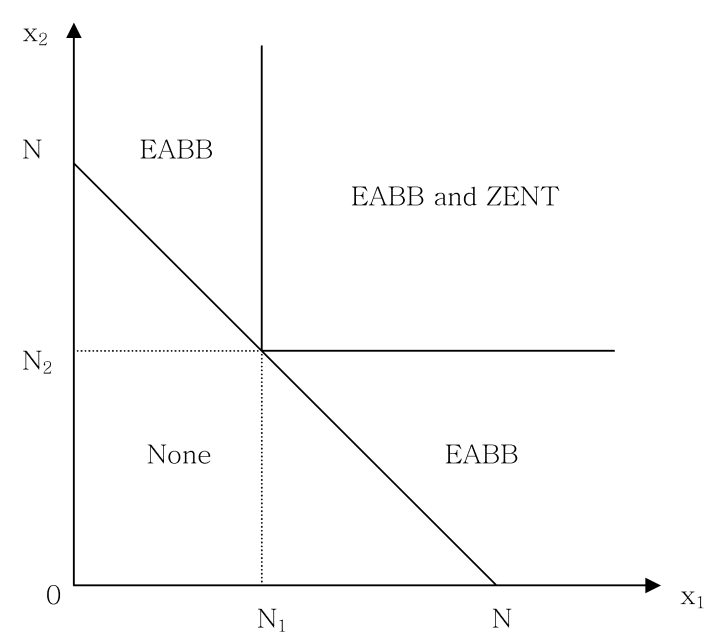

Fig 1.

From (7) and (8), we admit that not only the global consumption level is important, but also each country's consumption level must be large enough to match the condition for the existence of the international treaty. This observation matters for understanding the current trend of awareness on GPGs as each country's income increases.

On the other hand, the critical values representing the range of consumption levels are determined by the parameters of utility functions. Under preference uncertainty, the absolute level of private consumption is an important criterion for establishing an efficient international entity with incentive compatibility and individual rationality.

\subsection{Optimal/Universal Reservation Level}

The merit of our model and mechanism is that we could explain the co-existence of concessions and reservations in reality in the meaningful way. For example, we may explain why the implementation of the Kyoto Protocol gets lough as the number of the participating countries increases. As the countries with different preferences and resources get involved into the new expansion of the protocol at the stage of reservations, we would admit that the conservative concessions at the stage of concessions might be critical to remain as the agreement on the protocol.

There is an alternative way to explain concessions and/or reservations. That is to calculate, at one time, the optimal level of concessions in the framework of mechanism design. However, it is highly likely that there is usually the mixture of concessions and reservation in reality. 
Our interpretation of the reservations in international treaties has two concepts. On the one hand, related with Fon and Parisi (2003), there is an intra-country reservation, which is the level of openness to the international concessions. Our result would be directly concerned with that intra-country level of reservation in the model of a continuum of concession levels.

On the other, related with Barrett (2001), we may think of an inter-country reservation, which is a kind of "take it or leave" decision for each country. We could discuss a way of thinking our analysis to this inter-country reservation in the framework of two groups of countries with different income levels.

\section{(1) Optimal Reservation Level: Intra-country Reservations}

The reservation level at the stage of ratification is defined as

$$
r_{i}(\theta) \equiv \bar{z}_{i}(\theta)-z_{i}(\theta)=\frac{1}{p}\left(\bar{\theta}_{i}-\theta_{i}\right)
$$

where the first term, $\bar{z}_{i}(\theta)$, is given by the commitment of concessions and the second, $z_{i}(\theta)$, is the optimal level of concessions evaluated by the analysis.

The first term in (9) is the concession level in the treaty at the stage of concessions. It is just agreed at the most conservative stance. The second term is the final level of concessions in the treaty at the stage of ratification. It is agreed at the optimal level through negotiations. The difference $r_{i}(\theta)$ is the optimal reservation level, which would be observed in reality with variations from uncertain noisiness.

The important question in Fon and Parisi (2003) is that how to establish an incentive compatible mechanism to obtain the optimal level of reservations. Compared with Fon and Parisi (2003) with complete information, we consider incomplete information. Our analysis permits many countries more than two while their main concern is bilateral treaty contents.

\section{(2) The Universal Reservation: Inter-country Reservations}

The critical question in Barrett(2001) is that how does the introduction of side payments in the Montreal Protocol make it possible to establish a universal reservation and to promote to the formation of a universal agreement on a treaty. By using our analysis with a minor addition, we can discuss the meaning of Barrett(2001)'s conclusion. 
We assume that there are $l$ countries with the equation (8) being satisfied, $m$ countries with the equation (7) not being satisfied but the equation (8) being satisfied. Then, (i) it is possible that the former $l$ countries could form a treaty on concessions without the latter $m$ countries' participation. However, (ii) it is also possible that all the countries make a treaty on concessions with a possible later reservation.

There are several reasons for preferring the latter method. Firstly, the universal concessions with all the countries make it easy to introduce more concessions and reservations with transfers than a treaty of concessions with a small number of countries. Sunk in the treaty, the rich countries would listen to and meet the poor countries, and become more generous to the problems of the poor. Secondly, there would be an additional possibility of treaty in the sense of budget balance in the international entity. The universal treaty may promote the conventions and meetings among its member countries so as to increase the probability of the mechanisms with ex ante budget balancedness in (7) even when there is no possibility of the mechanisms with zero expected net transfer in (8).

\section{Conclusion}

We propose the optimal mechanism with monetary transfers by which we could understand international treaties over GPGs in the viewpoint of concessions and ratifications. Even though the mechanism could be finalized as the optimal level of contributions according to the mechanism with proportional division of the surplus in average, the decomposition of the contributions into concessions and ratifications could be supported both by the introduction of the Vienna Convention in the international society and by the new approach of the international arena for the universal participation over GPGs.

Theoretically speaking, we propose an alternative division method of the potential surplus from the international treaty over GPGs in contrast to the literature of Groves mechanisms. Our proportional division of the surplus summarized in the ZENT condition reflects a kind of fairness in the sense that the country with higher preference parameter values for global public goods may receive larger surplus in a proportional way eventually in average from the combination of allocations and transfers designed by the international entity. Thus, our method of surplus division satisfies a fairness concept which is highly regarded as goodness in the international society. Especially in the case of CO2-emission reductions, every country needs to be regarded as a sovereign entity which may receive fairly the proportional potential surplus from the international cooperation. 
This paper departs from Makowski and Mezzetti(1994) in that we assume the difference in type sets from country to country. Because of that difference of type sets, our ZENT condition becomes genuinely stricter than their EABB condition. And, our ZENT condition is meaningful in the international society with member countries which have sovereign powers.

This paper, on the other hand, suggests a seminal model of mechanism design for the cases of international treaties by introducing incomplete information on the characteristics or types among the member countries. Even though Fon and Parisi(2003) introduce concessions, ratifications, and reservations with a kind of economic model, their model is lack of uncertainty and deals with two nations. Our model in this paper lay a foundation for the general and unified framework to many GPGs including environmental issues, epidemic issues, and spillover issues. This new framework for incomplete information would be used for many cases of GPGs. For example, we may think of a regional problem with externality for several countries.

This paper has several limitations. Firstly, we select the outside option payoff as exogenous value and normalize it as 0 . Surely, the outside option payoff could be calculated endogenously in some cases. However, the calculation from the endogeneity of outside option in our setup is technically hard because of the diversity of type sets in international treaty-making environment for GPGs. Secondly, we introduce general case of $\mathrm{n}$ countries into our setup. More specific result would be possible for the case of regional public goods with several countries. Thirdly, we use quasi-linear welfare functions. More general functions could be the object of another paper. 


\section{References}

Barrett, S. (2001). International cooperation for sale, European Economic Review 45: $1835-1850$

Burniaux, J. M., Chateau, J., Dellink, R., Duval, R. and Jamet, S.(2009) The economics of climate change mitigation: How to build the necessary global action in a cost-effective manner, OECD

Dasgupta, P. S., Hammond, P. J. and Maskin, E.S.(1979). The implementation of social choice rules: Some general results on incentive compatibility, Review of Economic Studies 46: 185-216

Ferroni M. and Mody A.(2002). International Public Goods; Incentives, Measurement, and Financing, The World Bank

Fon V. and Parisi F.(2003). The Hidden bias of the Vienna Convention on the law of treaties, Law and Economics Working Paper Series 03-20, George Mason University

Groves, T. and Loeb M.(1975). Incentives and public inputs, Journal of Public Economics 4: $211-226$

Holmström, B. (1979). Groves schemes on restricted domains, Econometrica 47: $1137-1144$

Ihori, T. (1994). Strategic transfers and private provision of public goods, Journal of Public Economics 57: 489-505

Ihori, T. (1996), International public goods and contribution productivity differentials, Journal of Public Economics 61: 139-154

Kaul, I., Grunberg, I. and Stern, M.(1999). Global Public Goods: International Cooperation in the $21^{\text {st }}$ Century. New York and Oxford: Oxford University Press 
Laffont J.J. and Martimort D.(2005). The design of transnational public good mechanisms for developing countries, Journal of Public Economics 89: 159-196

Makowski, L. and Mezzetti C.(1994). Bayesian and weakly robust first best mechanisms: Characterizations, Journal of Economic Theory 64: 500-519

Sandler, T. (1997). Global Challenges: An Approach to Environment, Political, and Economic Problems, Cambridge, U.K.: Cambridge University Press 